\title{
Comparative study of attenuation and dispersion of different pressure disturbances propagating in aerosols
}

Riady Siswoyo Jo, Basil T. Wong, Anatoli Vakhguelt

- School of Engineering

Abstract

This study aims to investigate the attenuation and dispersion of pressure waves propagating in aerosols. A numerical scheme based on Crank-Nicolson finite differencing and trapezoidal quadrature discretization methods is employed to simulate the evolutions of several pressure waves with different initial profiles (disturbances). The attenuation and dispersion are observed from the spectra of the wave profiles at different times of propagation obtained by performing FFT (Fast Fourier Transform) to the simulated wave profiles. Simulation results show distinctive behaviors of different pressure disturbances propagating through the same medium.

Original language

Title of host publication

Publisher

Volume

ISBN (Electronic)

State

Event

Other

Country

City

Period
English

International Conference of Numerical Analysis and Applied Mathematics 2015, ICNAAM 2015

American Institute of Physics Inc.

1738

9780735413924

Published - Jun 82016

International Conference of Numerical Analysis and Applied Mathematics 2015, ICNAAM 2015 - Rhodes, Greece

International Conference of Numerical Analysis and Applied

Mathematics 2015, ICNAAM 2015

Greece

Rhodes

$9 / 23 / 15 \rightarrow 9 / 29 / 15$

Jo, R. S., Wong, B. T., \& Vakhguelt, A. (2016). Comparative study of attenuation and dispersion of different pressure disturbances propagating in aerosols. In International Conference of Numerical Analysis and Applied Mathematics 2015, ICNAAM 2015. (Vol. 1738). [030003] American Institute of Physics Inc.. DOI: $\underline{10.1063 / 1.4951759}$ 